The Astrophysical Journal, 474:518-528, 1997 January 1

(C) 1997. The American Astronomical Society. All rights reserved. Printed in U.S.A.

\title{
ULTRAVIOLET JETS AND BRIGHT POINTS IN THE SOLAR CHROMOSPHERE. II. STATISTICAL CORRELATIONS
}

\author{
N. M. HoEKzEMA AND R. J. RutTeN \\ Sterrekundig Institut, Postbus 80 000, NL-3508 TA Utrecht, The Netherlands \\ AND \\ J. W. CoOK \\ E.O. Hulburt Center for Space Research, Code 7668, Naval Research Laboratory, Washington, DC 20375-5352 \\ Received 1996 March 20; accepted 1996 July 24
}

\begin{abstract}
We use HRTS-VI rocket observations of the solar chromosphere to search for relationships between high-Dopplershift "jets" observed in the C I lines near $\lambda=156 \mathrm{~nm}$ and internetwork "bright points" observed in the $\lambda=160 \mathrm{~nm}$ continuum, in sequel to the analysis by Cook et al. which failed to find a direct connection between these phenomena. We now use the same data to establish statistical correlations between $C_{\text {I }}$ Dopplershift and $160 \mathrm{~nm}$ brightness modulation in internetwork areas. These mean relations emerge only after extensive spatial averaging and have small amplitude, but are definitely significant. They show that both C I Dopplershift and $160 \mathrm{~nm}$ brightness participate in oscillatory behavior with 3 minute periodicity and mesoscale $(8 \mathrm{Mm}$ wavelength) as well as small-scale (1.4 Mm wavelength) spatial patterning. We find spatial and temporal phase relations between Dopplershift and brightness that confirm that jets and bright points should not be interpreted as isolated entities. Rather, they are chromospheric manifestations, with much pattern interference, of the oscillatory acoustic shock dynamics in the internetwork which also cause $\mathrm{Ca}$ II $\mathrm{K}_{2 V}$ grains. Additional small-scale modulation is present which we attribute to waves with $f$-mode character.

Subject headings: Sun: chromosphere - Sun: oscillations - Sun: UV radiation
\end{abstract}

\section{INTRODUCTION}

In this paper we continue the search by Cook, Rutten, \& Hoekzema (1996, hereafter Paper I) for relations between C I jets and $160 \mathrm{~nm}$ bright points in the solar chromosphere, using ultraviolet spectrograms and spectroheliograms obtained with the HRTS rocket spectrometer. Paper I failed to find a direct relationship; here, we turn to statistical correlations between jets and bright points.

Chromospheric $\mathrm{C}$ I jets are defined as locations where the $C$ I resonance lines near $\lambda=156.1 \mathrm{~nm}$ show redshifted or blueshifted emission features with apparent Dopplershift exceeding $10 \mathrm{~km} \mathrm{~s}^{-1}$ (Dere et al. 1983, 1986) or $15 \mathrm{~km} \mathrm{~s}^{-1}$ (Paper I and this paper). Their spatial extent tends to be small, often of order $1^{\prime \prime}-2^{\prime \prime}$. Their characteristic lifetime is about $40 \mathrm{~s}$, sometimes up to $100 \mathrm{~s}$. They are predominantly present in quiet-Sun internetwork areas ("cell interiors"), avoiding the chromospheric network that appears bright in the $\mathrm{C}$ I lines. There are more blueshifted $\mathrm{C}$ I jets ("blue jets") than redshifted ones ("red jets").

The continuum around $\lambda=160 \mathrm{~nm}$ presumably originates in the layers around the temperature minimum between photosphere and chromosphere, according to the VALIII standard model of the solar atmosphere (Vernazza, Avrett, \& Loeser 1981). Filtergrams and spectroheliograms in this band, respectively taken with the Transition Region Camera and the HRTS slitjaw spectroheliograph, display bright network which consists of long-lived, closely packed grains, and dark internetwork areas in which more isolated bright grains appear and disappear. The latter are the $160 \mathrm{~nm}$ bright points discussed here. They have 50-100 K brightness temperature excess, lifetimes of about 1 minute and sizes of about $1^{\prime \prime}$. Their spatial density of 10-30 grains per cell interior is smaller than that of $\mathrm{C}_{\mathrm{I}}$ jets; the corresponding internetwork coverages are roughly $5 \%$ and $12 \%$, respectively (e.g., Bonnet et al. 1982; Cook, Brueckner, \& Bartoe 1983; Foing \& Bonnet 1984a, 1984b; Foing, Bonnet, \& Bruner 1986; Paper I).

As discussed in Paper I, it has been suggested that both the internetwork $\mathrm{C}_{\mathrm{I}}$ jets and the internetwork $160 \mathrm{~nm}$ bright points are related to the $\mathrm{Ca}$ II $\mathrm{H}_{2 V}$ and $\mathrm{K}_{2 V}$ internetwork grains (Bonnet et al. 1982; Cook et al. 1983; Foing \& Bonnet 1984b; Dere et al. 1986; Rutten \& Uitenbroek 1991a, 1991b). The nature of the $\mathrm{K}_{2 V}$ grains has in the meantime been clarified, in particular through the detailed numerical simulations of Carlsson \& Stein (1994). These grains are manifestations of shock formation in upward propagating acoustic waves with interference from backfalling matter from previous shocks. More detail is given in the reviews by Rutten \& Uitenbroek (1991a) and Rutten (1994, 1995). The conclusion is that the quiet-Sun internetwork chromosphere is pervaded by shocks to large extent, so much that even the existence of the standardmodel temperature minimum is in doubt (Carlsson \& Stein 1994, 1995).

Direct connections between $\mathrm{K}_{2 V}$ grains and $160 \mathrm{~nm}$ bright points or between $\mathrm{K}_{2 V}$ grains and $\mathrm{C}_{\mathrm{I}}$ jets have never been established, for lack of the required simultaneous and co-spatial ultraviolet and $\mathrm{Ca}$ II data sets of sufficient quality. The only published comparison, by Martić et al. (1991), indicates qualitative spatial similarity between $\mathrm{K}_{2 V}$ and 160 $\mathrm{nm}$ brightness structuring, but only after spatial smearing to $3^{\prime \prime}$ resolution that may largely be set by $\mathrm{K}_{2 V}$ seeing.

Our aim in these papers is therefore to wonder whether oscillatory relationships may be found between $160 \mathrm{~nm}$ bright points and $\mathrm{C}$ I jets alone, possibly with phase delays as suggested by Rutten \& Uitenbroek (1991b). Paper I searched for direct one-to-one relationships without finding any. In this paper we turn to statistical analysis of the same 
HRTS data set, correlating Dopplershift and brightness at all magnitudes rather than only the extreme excursions, and averaging the data spatially in order to suppress noise. Small-amplitude but significant relationships then emerge.

\section{INPUT DATA}

The observations used for this study are the same as in Paper I. They were obtained with the HRTS-VI rocket flight on 1988 November 20 and are briefly summarized here. Further detail is given in Paper I.

The $160 \mathrm{~nm}$ continuum data consist of seven spectroheliograms taken in an $1 \mathrm{~nm}$ passband centered at $\lambda=160$ $\mathrm{nm}$. They were exposed during $7.2 \mathrm{~s}$ at roughly $25 \mathrm{~s}$ intervals, spanning a duration of $160 \mathrm{~s}$. Meanwhile, the slit of the HRTS spectrograph was stepped across the field so that each pixel of the seven heliograms was sampled once for C I Dopplershift during this period.

Following Paper I, we limit the analysis to a $150^{\prime \prime} \times 87^{\prime \prime}$ subfield that is relatively free of blemishes and covers the seven $160 \mathrm{~nm}$ heliograms. It is shown in Figure 1 (Plate 9) (C I Dopplershift) and Figure 2 (160 nm brightness). The pixels are $0.5 \times 00^{\prime \prime} .5$. The spatial resolution is about $1^{\prime \prime}$, as is the precision of the alignment between the $\mathrm{C}$ I map and the $160 \mathrm{~nm}$ heliograms. The scanning for the Dopplershift map (upper panel of Fig. 1) was sequential with the slit oriented along rows; the top row was measured $160 \mathrm{~s}$ after the bottom row. The bottom and top parts were scanned with 2 " slit steps; the middle part in 1 " steps.

The lower panel of Figure 1 is an enlargement of the center part of the subfield. The preponderance of blue jets over red jets is evident. Most jets represent peaks: they lie in wider areas with smaller Dopplershift of the same sign (blue within green, red within yellow). This suggests an oscillatory pattern of which the jets mark wave extrema, rather than a flat background from which jets arise as isolated features.

The observed $160 \mathrm{~nm}$ brightness values were converted into brightness temperatures $T_{b}$ by assuming that the spatially and temporally averaged mean intensity equals the Planck function for $T=4300 \mathrm{~K}$, representative of the VALIII temperature minimum. This choice is somewhat arbitrary. The resulting brightness temperatures seem slightly lower and slightly higher, respectively, than the comparable scans in Cook et al. (1983) and Foing et al. (1986). However, our discussions below concern only differential $T_{b}$ changes.

The top panel in Figure 2 shows the selected subfield from the first of the seven heliograms. It displays the network as bright extended patches while the internetwork is filled with grainy brightness structure at various scales. Some of the fine-scale variation is due to film grain. The second panel, the same as Figure 2 of Paper I, shows the average of the seven heliograms. The averaging reduces the film grain noise as well as the internetwork brightness variation which varies considerably between heliograms. It enhances the network-internetwork contrast since the network is relatively stable. The third panel shows reverse patterning by displaying the rms brightness temperature variation $\sigma T_{b}$ per pixel over the seven heliograms. In this representation the more stable network tends to be dark, while the internetwork regions display patchy clustering with blobs of 4 " $-10^{\prime \prime}$, corresponding to the " $8 \mathrm{Mm}$ wavelength scale" discussed by Foing \& Bonnet (1984a), Foing et al. (1986), Martić \& Damé (1989), and Cook \& Ewing (1991). It is enhanced in the bottom panel of Figure 2 by
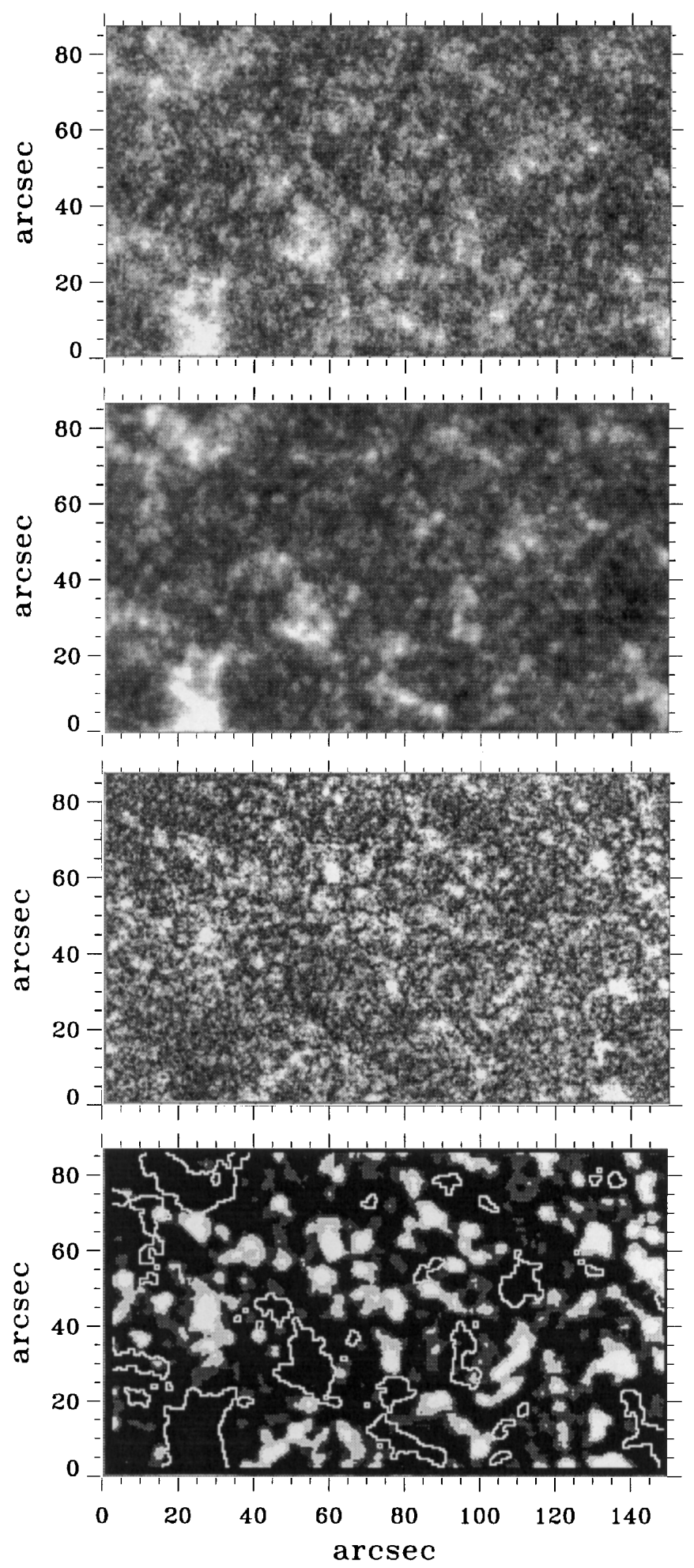

FIG. 2.-Four $160 \mathrm{~nm}$ brightness maps for the $150^{\prime \prime} \times 87^{\prime \prime}$ subfield. Top panel: the first of the seven heliograms, taken at $t=199 \mathrm{~s}$ after launch. The chromospheric network stands out as large bright patches, while internetwork areas contain clusters of bright points of varying size and intensity. Second panel: temporal average of the seven heliograms, covering $160 \mathrm{~s}$. The steady network pattern gains contrast while the short-lived internetwork bright points and noise from film graininess cancel out. Third panel: rms temporal brightness variation $\sigma T_{b}$ per pixel over the seven heliograms. The network shows appreciably smaller temporal variability than the internetwork regions. The latter show much spatial variation in temporal variability, with clumpy patterning. Bottom panel: spatially smoothed rms brightness variation, using coarse brightness binning to emphasize meso-scale patterning. The overlaid contours outline the network mask used throughout the paper. 
spatial smoothing plus coarse brightness binning. The third panel also shows substantial brightness variation at much smaller spatial scales, close to the $1^{\prime \prime}$ resolution limit and corresponding to the $1.4-3 \mathrm{Mm}$ characteristic bright point separation noted by Foing \& Bonnet (1984a, 1984b) and Foing et al. (1986).

The bottom panel of Figure 2 displays the network mask to restrict the analysis below to internetwork alone. It cuts out all pixels in the second panel that are over $10 \%$ brighter than the spatial mean. These areas are colored gray in Figure 1.

The color black in Figure 1 marks special locations where the HRTS data-analysis algorithm was not able to assign a Dopplershift to a $\mathrm{C}$ I emission feature by taking its first moment. For extended black areas (upper panel), such failure is generally due to lack of signal. Most of the smallscale black areas in the lower panel lack well-defined peaks in their C I profile (Paper I). These tend to lie within blue areas, suggesting that their nonmeasurability goes together with bluelike properties. Such black-near-blue pixels are treated below as a special Dopplershift class, and indeed turn out to behave generally as blue pixels - or even bluer than blue ones. With this in mind, the Dopplershift map also indicates split-scale patterning reminiscent of the 8-1.4 Mm spatial dichotomy of Foing \& Bonnet (1984a).

\section{STATISTICAL CORRELATIONS}

When searching for solar relationships between brightness and intensity, one tends either to analyze data in the spacetime domain, searching for coincidence between characteristic structures or events, or to turn to Fourier methods to disentangle multiple oscillatory components. In Paper I the first approach was tried by defining bright points and blue jets as specific entities. The logical alternative is to try the Fourier domain. However, each location in the C I Dopplershift map has been measured only once, whereas the seven $160 \mathrm{~nm}$ heliograms represent coarse temporal sampling of brief duration (160 s). Thus, Fourier analysis is not feasible on these rocket data and has to await longer duration SUMER sequences.

However, a third tack is feasible thanks to the absence of atmospheric seeing in space. The $C_{\text {I }}$ Dopplershift map and the $160 \mathrm{~nm}$ heliograms are spatially aligned to about the resolution limit. Since there are no larger scale timedependent "rubber sheet" geometrical deformations as in ground-based observing, pixel-by-pixel comparisons may be averaged to diminish the confusion that may arise from instrumental noise and solar interferences. Such selective averaging constitutes our approach below.

\subsection{Distributions}

Figure 3 details the relationship between time-averaged $160 \mathrm{~nm}$ brightness $T_{b}$ and temporal variability $\sigma T_{b}$ that is seen qualitatively by comparing the second and third panels of Figure 2. The scatter cloud has upward and rightward tails made up by dark to medium bright but variable internetwork pixels and by very bright but time-constant network elements, respectively. The mean curve in the bottom panel has a slope change at the network cutoff value. The right-hand end at $\sigma T_{b} \approx 10 \mathrm{~K}$ is about equal to the lower boundary of the scatter cloud and marks the rms variation of the brightest network grains. Since these are stable, the value indicates the noise level due to emulsion grain. The $10 \mathrm{~K}$ value [min-max excursions $2(2)^{1 / 2} \sigma T_{b} \approx 30$

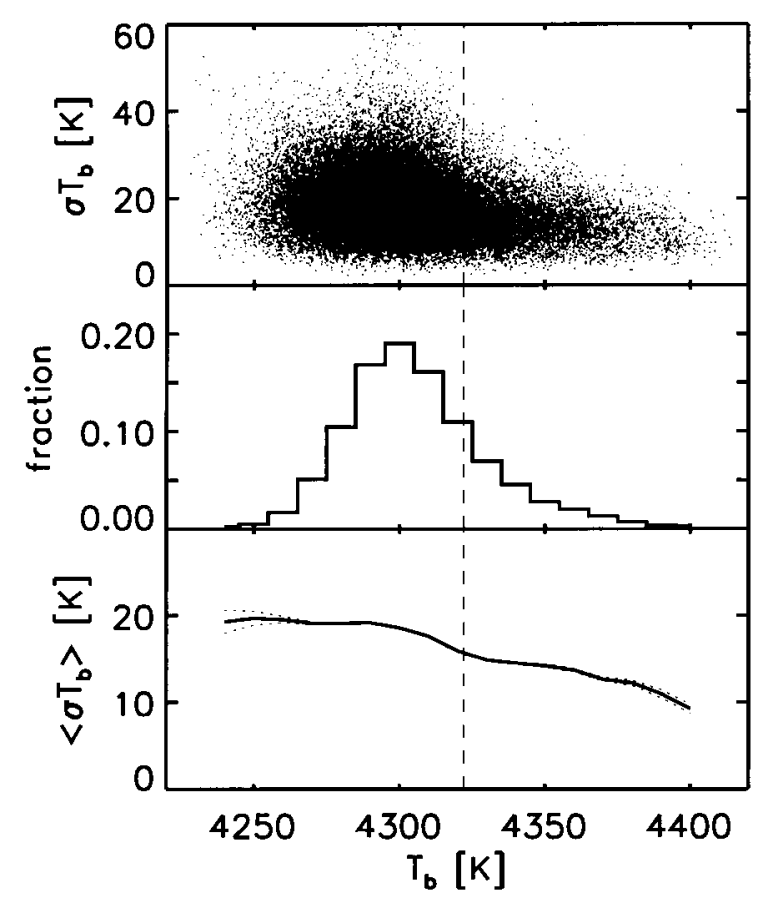

FIG. 3.-Brightness temperature variability $\sigma T_{b}$ against time-averaged brightness temperature $T_{b}$ per pixel. The dashed line defines the cutoff used to mask out the network (brighter). Upper panel: pixel-by-pixel scatter diagram. Middle panel: histogram of the pixel fraction per $T_{b}$ bin. Bottom panel: bin averages on an expanded vertical scale, with $1 \sigma$ variances (dotted curve).

$\mathrm{K}$ ] is similar to the emulsion noise in other HRTS results and the TRC data of Foing \& Bonnet (1984b). The largest values of $\sigma T_{b}$ lie in the internetwork domain left of the dividing line. They reach up to $\sigma T_{b} \approx 60 \mathrm{~K}$ or about $170 \mathrm{~K}$ brightening. The mean internetwork variation $\sigma T_{b} \approx 20 \mathrm{~K}$ is twice the noise.

Figure 4 shows the same quantities plotted against $\mathrm{C}_{\text {I }}$ Dopplershift and excluding network. The two scatter plots do not show correlation, but mean relationships appear in the lower panels by averaging per Dopplershift bin. Their amplitudes are much smaller than the spread in the upper panels (note the vertical scale expansions), but the symmetry of the two curves and their roughly parabolic shape suggest that, on the average, $160 \mathrm{~nm}$ brightness and C I Dopplershift are related to each other. These average correlations could be partially due to nonexcluded network pixels, but they remain present in tests (not shown) with lower temperature network cutoffs. The time-delay statistics discussed below add significance to their interpretation as an internetwork characteristic.

Figure 5 shows Dopplershift statistics. The solid curve in both panels specifies the internetwork surface fractions of the color-coded areas in Figure 1. It quantifies that blue jets $\left(\Delta \lambda_{\mathrm{D}}>15 \mathrm{~km} \mathrm{~s}^{-1}\right)$ are more numerous than red jets $\left(\Delta \lambda_{\mathrm{D}}<\right.$ $\left.-15 \mathrm{~km} \mathrm{~s}^{-1}\right)$ as noted by Dere et al. $(1983,1986)$ and also seen in Figure 4 of Paper I.

The dotted curves similarly specify Dopplershift distributions, but only for "bright points" by spatial averaging over those pixels that show large temporal $160 \mathrm{~nm}$ brightness variation. The left-hand panel specifies the Dopplershift distribution of pixels with $32<\sigma T_{b}<43 \mathrm{~K}$. More precisely, we selected the pixels with $90<2(2)^{1 / 2} \sigma T_{b}<120 \mathrm{~K}$, corresponding to a statistical min-max change in this range; this definition was used rather than the actual measured differ- 


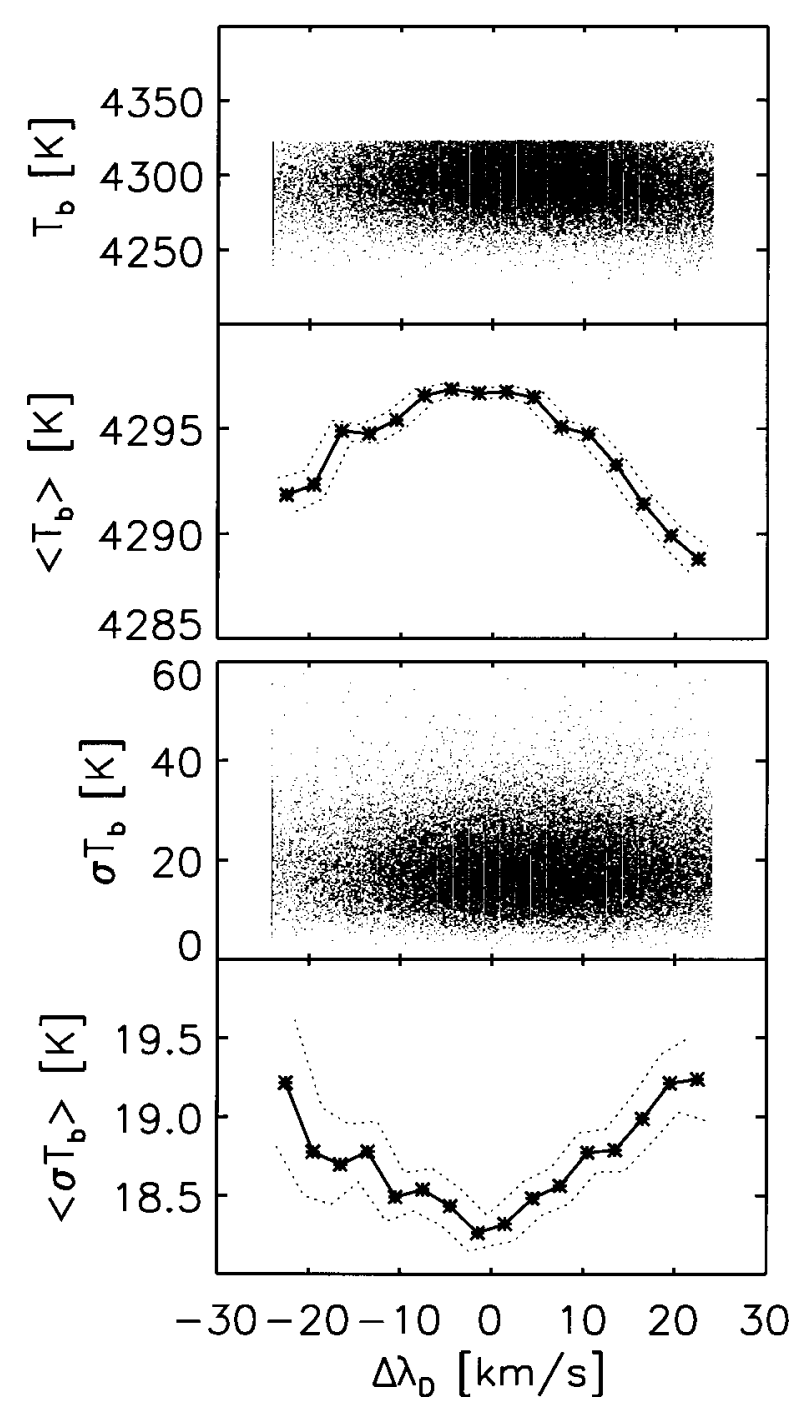

FIG. 4.-Statistical relations between brightness properties per pixel (vertical) and Dopplershift $\Delta \lambda_{\mathrm{D}}$ per pixel (horizontal). The upper graph is for time-averaged brightness temperature $T_{b}$; the lower graph for temporal brightness variability $\sigma T_{b}$. The scatter plots in the upper panels are cut at $\Delta \lambda_{\mathrm{D}}= \pm 24 \mathrm{~km} \mathrm{~s}^{-1}$ by the measurement algorithm; the upper scatter cutoff in the top panel is due to the exclusion of network pixels with $T_{b}>4322 \mathrm{~K}$. The lower panels show averages per $3 \mathrm{~km} \mathrm{~s}^{-1}$ Dopplershift bins, on much expanded vertical scales. The variances are standard deviations of the bin averages. ence $T_{b}^{\max }-T_{b}^{\min }$ per pixel in order to reduce noise, particularly from darkenings when the spectrograph slit passed over the pixel. This selection makes up $4 \%$ of the total area (nonblack and nongray in Fig. 1). The right-hand panel is for pixels with $\sigma T_{b}>43 \mathrm{~K}$, with a filling factor of only $0.4 \%$. The squares result when black pixels that are adjacent to blue jets are included in the blue-jet bin.

The dotted distribution at left is similar to the general distribution, except for the two extreme Dopplershift bins which are favored by large-variability pixels. These bins show substantial enhancement at right, especially when the black-near-blue pixels are added to the blue-jet bin (square at top right). Both extreme Dopplershift bins then contain twice the fraction of large-variability pixels that the general distribution has, at the cost of low-Dopplershift contributions. Thus, pixels with excessive $160 \mathrm{~nm}$ brightness variation lie preferentially at $C_{I}$ jet sites.

Note that even if all extreme $160 \mathrm{~nm}$ brightenings actually coincide with a $\mathrm{C}$ I jet location (simultaneously or at some phase lag), these distributions would not reach unity because the Dopplershift of each location was sampled only once. If all excessive $\Delta T_{b}$ locations mark bluejet locations at some phase delay, the single spectrograph scan would have missed many of the latter by sampling other C I Dopplershift phases.

\subsection{Temporal Relations}

Having found that, statistically, excess $160 \mathrm{~nm}$ variability correlates with excess $C_{\text {I }}$ Dopplershift, we now turn to the question of temporal phase delay. Our tactic is again to spatially average over bins of pixels with specific properties. Since the C I Dopplershift was measured only once per pixel, simultaneously along rows in Figure 1 and sequentially from bottom to top, we use the seven $160 \mathrm{~nm}$ heliograms to provide temporal variations with phase fixed to the moment of Dopplershift sampling. We have combined narrow strips from the heliograms into a single map that shows the $160 \mathrm{~nm}$ brightness per location within $15 \mathrm{~s}$ of the moment $t=t_{0}$ of its Dopplershift sampling. Similarly constructed maps specify $160 \mathrm{~nm}$ intensity per pixel at times 26 , $52,78,104$, and $130 \mathrm{~s}$ before and after the corresponding $\mathrm{C}_{\mathrm{I}}$ Dopplershift was measured. From these, we measured 160 $\mathrm{nm}$ brightness behavior per pixel as a function of positive or negative time lag with regards to the Dopplershift sampling.

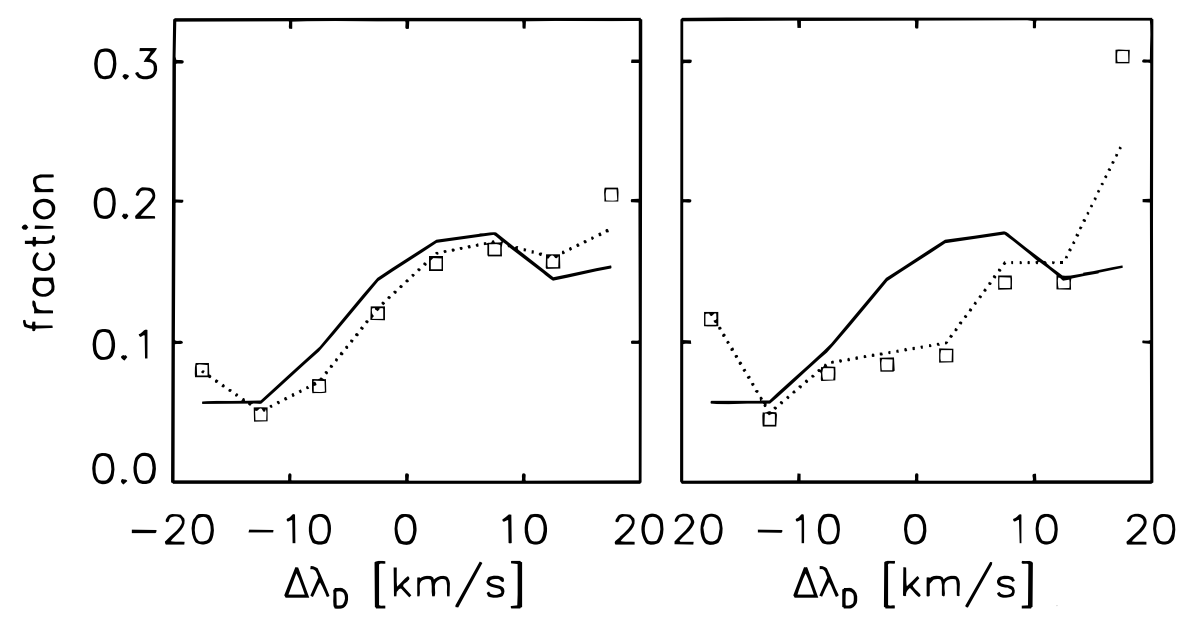

FIG. 5.-Bright point statistics. Solid curve: distribution over C I Dopplershift for all colored (nonblack and nongray) pixels in Fig. 1. Dotted curve: Dopplershift distributions for locations with large $160 \mathrm{~nm}$ brightness variability only. Left: locations with $32<\sigma T_{b}<43 \mathrm{~K}$. Right: locations with $\sigma T_{b}>43$ $\mathrm{K}$. The squares result when black pixels in Fig. 1 that are adjacent to blue jets are also included as blue jets. 
The time delay axis then covers $260 \mathrm{~s}$ duration. Pixels at the top of the C I map in Figure 1 furnish $130 \mathrm{~s}$ of $160 \mathrm{~nm}$ brightness history, while the brightness of pixels at the bottom of the C I map are followed for $130 \mathrm{~s}$ after their Dopplershift sampling. The results are shown in Figures 6-8.

In Figure 6 the time-delay bins are further split according to $C$ I Dopplershift. The curves show the spatial average of the $160 \mathrm{~nm}$ brightness evolution $\Delta T_{b}(t) \equiv T_{b}(t)-T_{b}$ of all pixels for which a given Dopplershift was measured when that pixel was sampled. Thus, the upper curve shows the

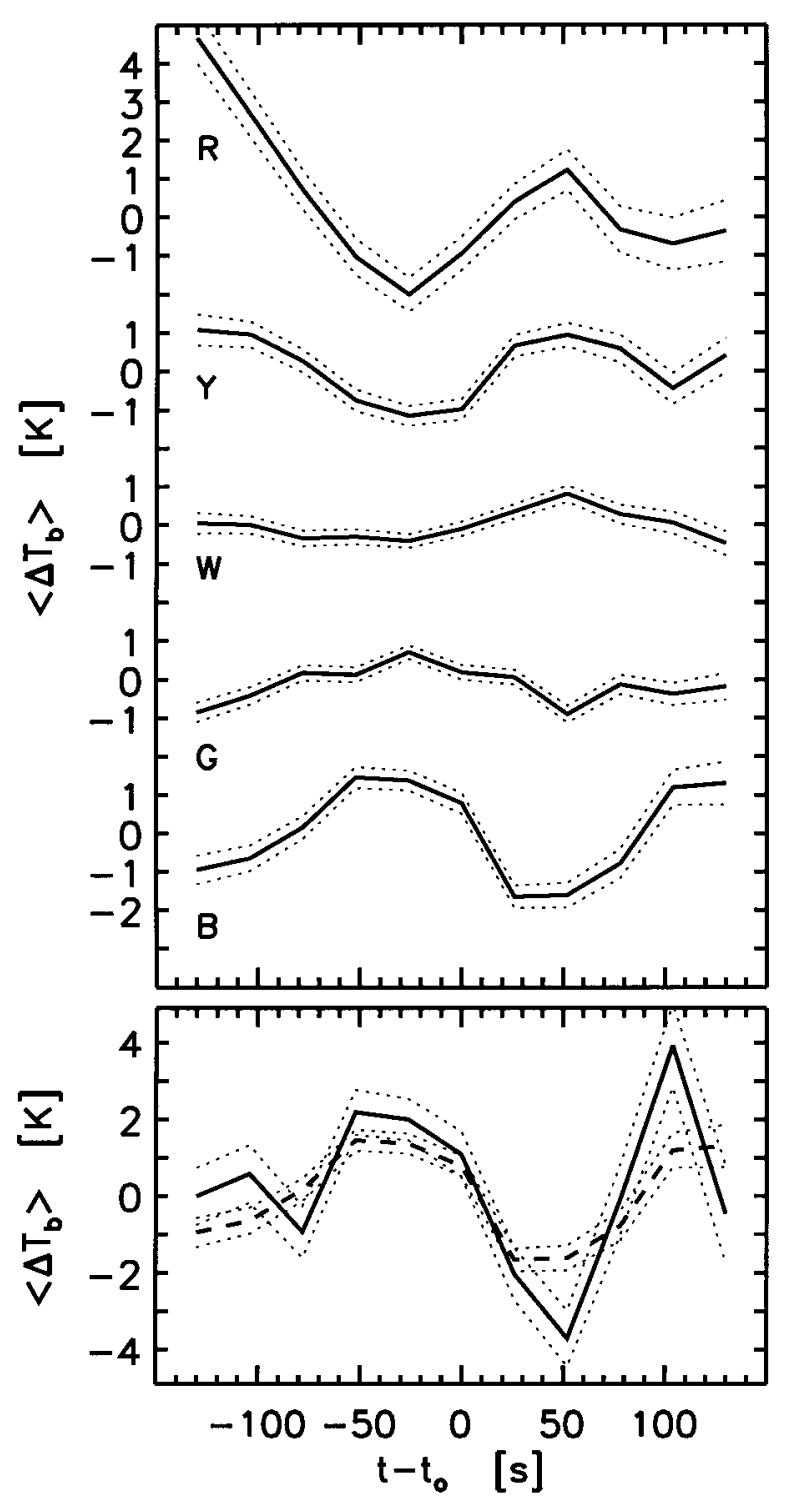

FIG. 6.-Time delay statistics. The abscissa measures the temporal separation $t-t_{0}$ between the C I Dopplershift measurement of a pixel at time $t=t_{0}$ and its brightness measurement from one of the seven $160 \mathrm{~nm}$ heliograms. Negative values imply brightness sampling before Dopplershift sampling. The ordinate is the corresponding difference between the instantaneous $160 \mathrm{~nm}$ brightness of a pixel and its time-averaged value, spatially averaged per time delay bin over all pixels per Dopplershift bin. The latter bins are coded by letters according to the color coding in Fig. 1. The dotted curves show the variances of the bin averages. A smallamplitude but significant brightness modulation appears, with 3 minute periodicity and opposite phase between red jets (top curve) and blue jets (bottom curve). The lower panel is a similar plot for black pixels with a blue-jet neighborhood (solid curve). The blue-jet curve is added for comparison (dashed curve).
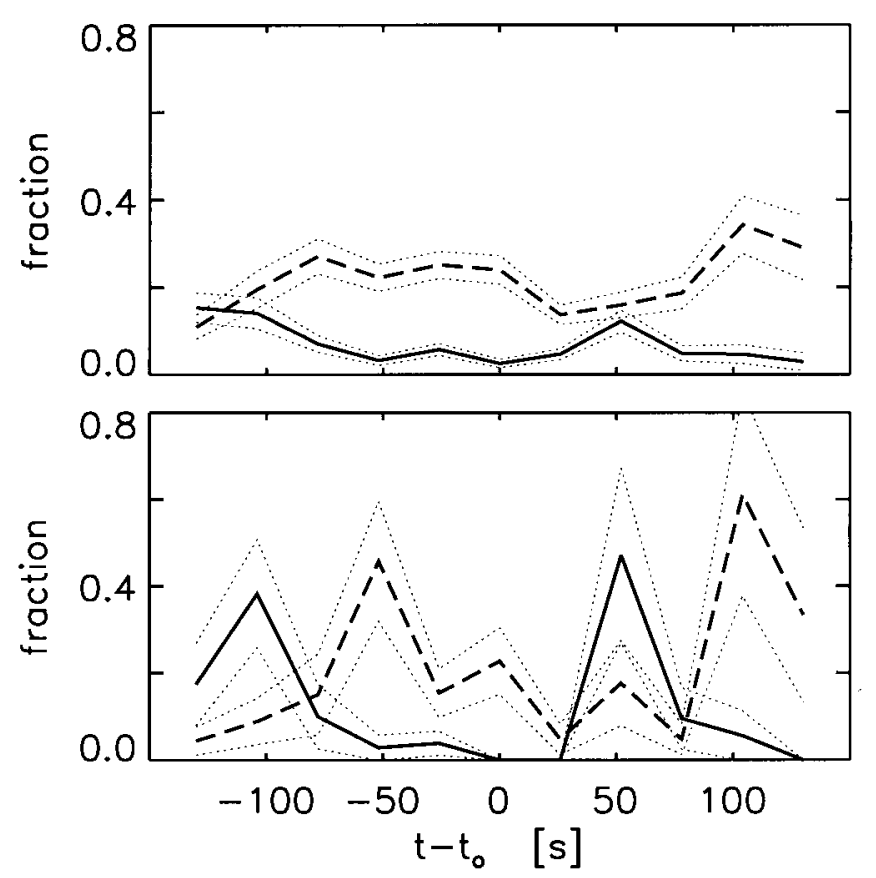

Fig. 7.-Time delay statistics for bright points only. Upper panel: bright points defined as internetwork pixels that at time $t$ were $45<$ $\Delta T_{b}<60 \mathrm{~K}$ brighter than their temporal mean. Lower panel: bright points defined as internetwork pixels that at time $t$ were $\Delta T_{b}>60 \mathrm{~K}$ brighter than their temporal mean. The abscissae again measure the temporal separation between $160 \mathrm{~nm}$ brightness sampling and $C_{\text {I }}$ Dopplershift sampling. The distributions measure the fraction of all such bright point pixels per time delay bin that coincide with a red jet at $t=t_{0}$ (solid curves) or with a blue jet or black-near-blue pixel at $t=t_{0}$ (dashed curve). Especially the extreme brightenings concentrate at specific lags prior to or after blue jets and red jets.

brightness prior to, at, and after the moment of Dopplershift sampling, averaged over all pixels at which a red jet was measured. The curve displays small-amplitude but significant modulation, far larger than the variance around the mean curve. The modulation implies that pixels with a red jet at $t=t_{0}$ are, on average, slightly brighter 2 minutes before the jet sampling, darker half a minute before the jet, brighter a minute after, and then darken again. In contrast, blue jets (lowest curve) produce reversed time modulation, with average brightening during the minute up to the jet, and darkening for about a minute after the jet. This agrees with the visual correlation noted from Figure 3 in Paper I, in which blue jets seem to favor dark regions of the $160 \mathrm{~nm}$ heliograms at a phase lag of about half a minute.

Together the red-jet and blue-jet curves suggest the presence of wavelike modulation with about 3 minute periodicity and $180^{\circ}$ phase shift between red and blue. The intermediate curves $(\mathrm{Y}, \mathrm{W}$, and $\mathrm{G}$ for yellow, white, and green Dopplershift bins, corresponding with the color coding of Fig. 1) display corresponding phase patterns but decreasing amplitude for decreasing Doppleshift size. This reduction may be partially due to phase mixing, since measuring small Dopplershift from a pixel that takes part in a regular Dopplershift oscillation does not discriminate between the rising and descending branches. Only the extreme Dopplershift values sample the phase of such oscillations precisely.

The bottom panel of Figure 6 shows similar time-delay modulation statistics for "black-near-blue" pixels. The blue jet curve from the upper panel is added for reference (dashed 

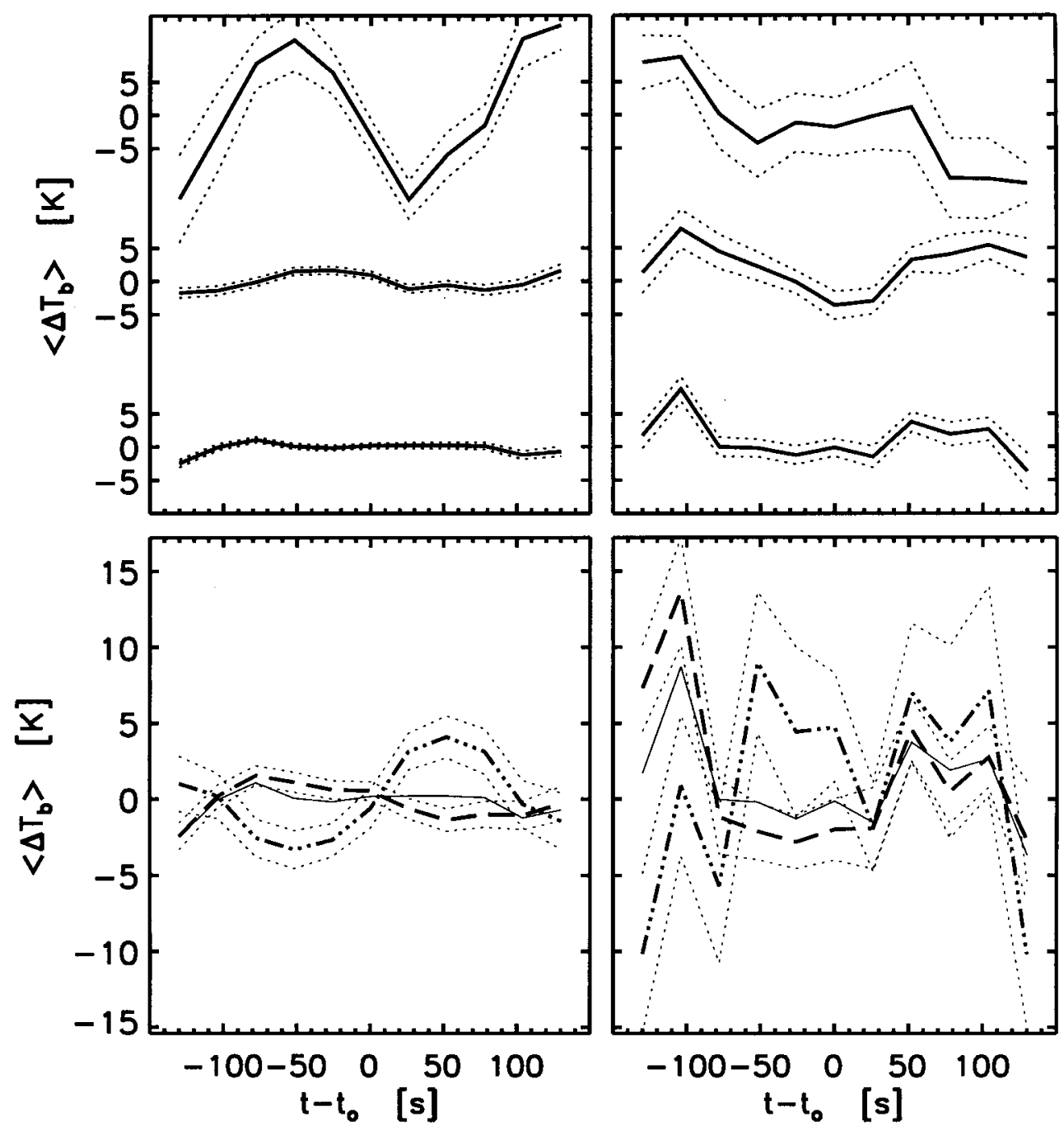

Fig. 8. - Time delay statistics as in Fig. 6 , split for jet geometry. The abscissae again measure time delay between the Dopplershift sampling at $t=t_{0}$ and the $160 \mathrm{~nm}$ brightness sampling. The ordinates again measure the instantaneous $160 \mathrm{~nm}$ brightness of a pixel minus its time average. However, the spatial averaging is now done over jet-geometry subclasses. The upper left panel is for blue jets. The top curve is for their centers, the middle curve for the remaining blue-jet pixels, the bottom curve for blue-jet perimeters. The upper right panel is similarly split for red jets. The bottom panels, also for blue jets (left) and red jets (right), split the spatial averaging over perimeter pixels between those with high Dopplershift (dashed curve; $10<\left|\Delta \lambda_{\mathrm{D}}\right|<15 \mathrm{~km} \mathrm{~s}^{-1}$ ) and low Dopplershift (dot-dashed curve; $0<\left|\Delta \lambda_{\mathrm{D}}\right|<3 \mathrm{~km} \mathrm{~s}^{-1}$ ). The thin solid curves are for all perimeter pixels (same as bottom curves in upper panels). The dotted curves show the variances of the averages. For blue jets the correlation between brightness modulation and Dopplershift drops off steeply away from jet center, with sign reversal for low-Dopplershift perimeters.

curve). The solid curve displays similar 3 minute modulation with similar phase at somewhat larger amplitude. Thus, these locations with complex $\mathrm{C}$ I profiles seem to behave as blue (or bluer than blue) jets.

These results may also be cast in the form of Figure 5 by plotting time-delay statistics for only those $160 \mathrm{~nm}$ pixels that show excessive brightness variations. This is done in Figure 7, showing that $160 \mathrm{~nm}$ bright points adhere to the modulations seen in Figure 6. The solid curve in the bottom panel of Figure 7 indicates that red jets are never exceptionally bright at the very moment, but that about $100 \mathrm{~s}$ earlier and $50 \mathrm{~s}$ later, their locations make up about $40 \%$ of all 160 $\mathrm{nm}$ pixels that show large temporary brightening at these time delays from the Dopplershift measurement. Reversely, about half of the extreme $160 \mathrm{~nm}$ brightenings which occur $50 \mathrm{~s}$ before or $100 \mathrm{~s}$ after the Dopplershift was sampled had blue-jet or black-near-blue Dopplershift at the moment of sampling, 2-3 times the normal surface fraction covered by these Dopplershifts (solid distribution in Figure 5).

\subsection{Spatial Relations}

The increase of modulation amplitude from blue-jet pixels to black pixels in Figure 6 indicates that the centers of blue jet areas may possess the largest average brightness modulation. We have therefore split the jet areas geometrically into jet centers, defined as jet pixels that are fully surrounded by other jet pixels $\left(\Delta \lambda_{\mathrm{D}}>15 \mathrm{~km} \mathrm{~s}^{-1}\right)$, and the remaining jet areas. This split produces the average relations in Figure 8. Spatial time-delay averaging over blue jet-center pixels alone (top curve in upper-left panel) indeed produces appreciably larger 3 minute modulation than for the remaining blue jet pixels (second curve, modulation amplitude similar to the overall blue-jet curve in Fig. 6). For red jets (right-hand panels) the concentration of modulation amplitude toward the jet center is less outspoken.

The bottom curves in the top panels and the bottom panels of Fig. 8 concern jet perimeters, being the first pixels outside a jet area. In the upper panels, these behave qualit- 
atively as the noncenter jet pixels (middle curves). In the lower panels, the perimeter-pixel averaging has been split again, now between relatively high Dopplershift (dashed curve) and low Dopplershift (dot-dashed curve) areas, with the complete sample shown for reference (thin solid curve). The right-hand panel has larger variances due to the smaller number of red-jet pixels. The left-hand panel shows significant differences in both average phase and average modulation between low and high Dopplershift perimeters. The averaged low-Dopplershift perimeter modulation varies in counterphase to the averaged blue-jet center modulation, whereas the averaged high-Dopplershift modulation appears to be in phase. The red-jet panel, though noisy, suggests similar phase behavior. Thus, the averaged correlation between brightness modulation and Dopplershift decays fast with separation from jet center, especially for blue jets. For these, the modulation flips phase already one 0.5 pixel $^{-1}$ outside the jet when the Dopplershift there is small.

The spatial decay of the averaged correlations around jet areas is studied further in Figure 9. The seven-heliogram averaged brightness temperature per pixel $T_{b}$ and the averaged variability $\sigma T_{b}$ that were binned for Dopplershift in Figure 4 are now binned for jet geometry. The upper panels show that on average, both blue jets (triangles) and red jets (squares) are darker than their surroundings but show larger brightness variability at $160 \mathrm{~nm}$. The left-hand panel indicates a significant difference between red and blue jets, the latter being darker.

The lower panels of Figure 9 result from averaging exclusively over areas in and around jets which contain jet centers. The variances are therefore larger than in the top panels, but the correlations are nevertheless well defined, which implies that selecting these jets enhances the selection of specific horizontal patterning. The latter differs strikingly from the correlations in the top panels, particularly for the $\sigma T_{b}$ panel at right in which the two curves display opposite behavior with enhanced variability close to blue jets and reduced variability near red jets. The split between the curves starts already within the jets $(\Delta r=-1)$ and extends to $\Delta r \approx 3$ over 4 pixels, corresponding to $2^{\prime \prime}-3^{\prime \prime}$ spatial distance (pixel diameters being 0.5-0.7 depending on direction).

The pattern suggests spatial periodicity with this wavelength. Such small-scale periodicity may, if present, show up also in the images themselves. Tests for $2^{\prime \prime}-3^{\prime \prime}$ spatial periodicity in the $160 \mathrm{~nm}$ heliograms are hampered by the emulsion graininess, but the elaborate analysis of Foing \& Bonnet (1984a) gave evidence for $1.4 \mathrm{Mm}\left(2^{\prime \prime}\right)$ preferred bright-point separation. A similar test for spatial periodicity in the C I Dopplershift map is shown in Figure 10. It results from an analysis in which sites with extreme Dopplershifts were selected that are well apart. Pixel rings were cast around them with increasing radius; for each, the surface
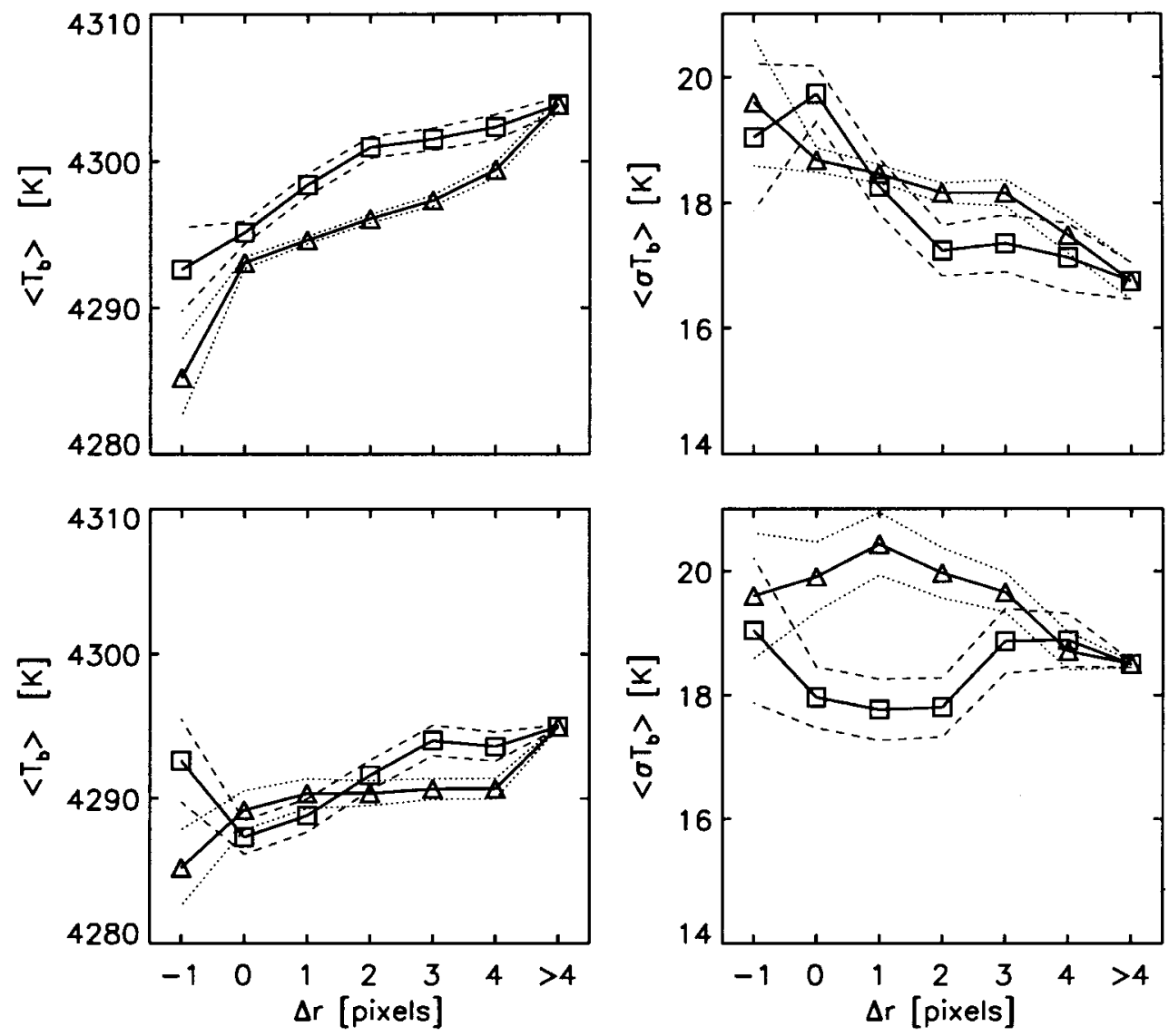

Fig. 9.- Statistical relations between $\mathrm{C}_{\mathrm{I}}$ Dopplershift and $160 \mathrm{~nm}$ brightness for blue jets (triangles) and red jets (squares). The abscissae measure the distance to jet center areas of successive pixel rings around jets, in pixel widths with $\Delta r=-1$ denoting jet center pixels, $\Delta r=0$ the remaining jet pixels, $\Delta r=1$ jet perimeter pixels, $\Delta r=2-4$ pixels at that distance from a jet, and $\Delta r>4$ all remaining pixels. The left-hand panels measure mean pixel brightness temperature $T_{b}$, temporally averaged over the seven $160 \mathrm{~nm}$ heliograms and spatially averaged over all pixels per bin. The right-hand panels measure temporal brightness variability per pixel, similarly averaged spatially. In the lower panels the spatial averaging is limited to only those jets that possess jet centers. The dotted curves are average variances. These plots suggest the presence of small-scale (3-4 pixel) spatial modulation. 


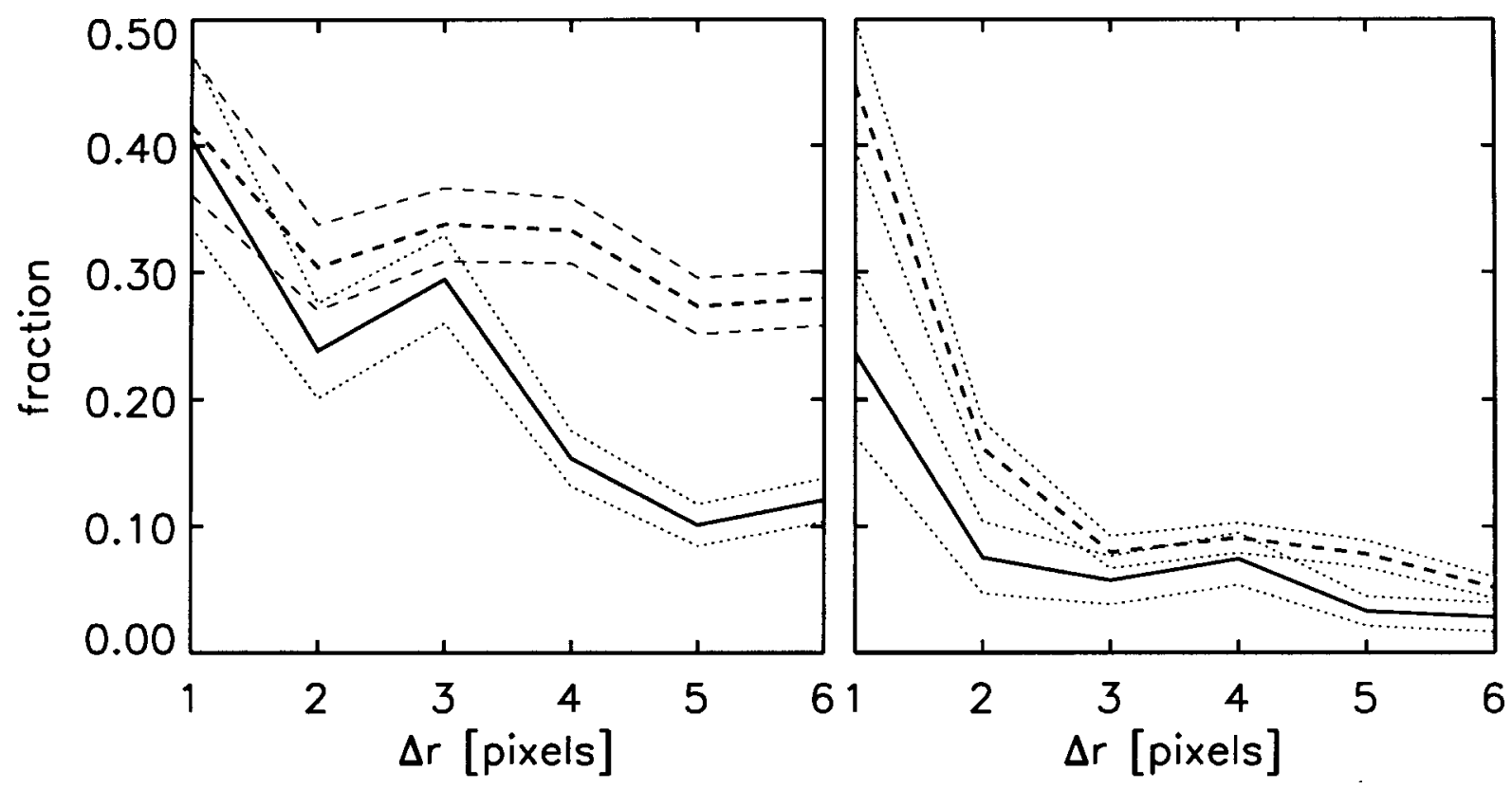

FIG. 10. - Jet separation statistics, respectively for blue jets (left) and red jets (right). For each type, well-separated jets were selected with $\left|\Delta \lambda_{\mathrm{D}}\right| \geq>24 \mathrm{~km}$ $\mathrm{s}^{-1}$ (the outer limits in Fig. 4) with two subfields. The curves measure, for each field separately (solid and dashed curves), the surface fraction of the pixels around the jets with $\left|\Delta \lambda_{\mathrm{D}}\right|>21 \mathrm{~km} \mathrm{~s}^{-1}$ as a function of the distance to the jet. The variances are Poisson estimates. The humps suggest preference for 3-4 pixel separations between jets.

fraction occupied by pixels with Dopplershift $\left|\Delta \lambda_{\mathrm{D}}\right|>21$ $\mathrm{km} \mathrm{s}^{-1}$ of the same sign was measured. This was done for two vertically narrow subfields, the first being the one shown in the lower panel of Figure 1, the other one outside the area shown in the upper panel (and without $160 \mathrm{~nm}$ coverage). The resulting curves in Figure 10 differ appreciably between the two subfields, by more than the Poisson variance estimates. Nevertheless, the curves show humps that indicate a preference for jet repetition at 3-4 pixel separation (corresponding to $1^{\prime \prime} .8-2$ ".4). This result agrees with the impression from the lower panel of Figure 1 that many jets possess split centers.

\section{DISCUSSION}

The diagrams above show relationships between $\mathrm{C}$ I Dopplershift and $160 \mathrm{~nm}$ brightness that are statistically significant. The key features are the skewedness of the bright-point distribution with Dopplershift (Fig. 5), the opposite red and blue modulations in Figure 6, the preference of the largest brightness changes in Figure 7 to occur at specific phases of the Dopplershift modulation, the spatial scalings in Figures 8-10, and the spatially averaged morphological differences between red and blue jets in Figures 8-9. Altogether, they indicate the existence of oscillatory phenomena in internetwork regions, with about 3 minute temporal periodicity and $2^{\prime \prime}-3$ " horizontal scaling, in which both signals participate with definite phase relationships beween them.

Obviously, these modulations are reminiscent of the chromospheric 3 minute oscillation known from the $\mathrm{Ca}$ II $\mathrm{H}$ and $\mathrm{K}$ lines and other diagnostics, also a small-scale internetwork phenomenon (see reviews by Rutten \& Uitenbroek 1991a; Rutten 1994, 1995). However, while significant within their statistical variances, the modulations do not imply one-to-one feature-by-feature relationships as searched for in Paper I. The bottom panel of Figure 7 shows that half of the $160 \mathrm{~nm}$ brightenings precede or follow blue jets or red jets at specific time lags, but that plot holds only for the most exceptional brightenings. Their internetwork area filling factor at any one moment is less than $0.5 \%$. The 45-60 $\mathrm{K}$ brightenings in the upper panel make up only $1 \%-2 \%$ of the area; for them the jet-correlated modulation drops already to about $20 \%$.

Our finding of statistical correlations at small amplitude suggests that either only a few exceptional bright points and jets alternate in such phase-delay patterns or that more global oscillatory relationships are heavily masked by other phenomena. We have tested the first possibility by replotting the distributions in Figures 6 and 7 after deleting the pixels with the largest $\Delta T_{b}$, with various cutoffs. We found that the correlations in the upper panel of Figure 7 lose amplitude for lower cutoff but remain significantly present, while the modulations in Figure 6 diminish only marginally. These tests indicate that the brightest points (excessive $\Delta T_{b}$ ) mark extremes of modulations that are present also more generally, as is also indicated by the small variances in Figure 6 . This implies considerable masking by measurement noise and solar interference.

\subsection{Noise and Interference}

The right-hand end of the mean curve in the second panel of Figure 3 indicates a noise limit of about $\sigma T_{b} \approx 10 \mathrm{~K}$ or excursions of order $30 \mathrm{~K}$. This noise is mostly due from emulsion grain. Its contribution is largest at small scales so that our spatial averaging reduces its effect. The C I Dopplershift measurement is less sensitive to film grain because it uses the full line profile.

Other instrumental noise comes from variations in heliogram image quality. The spectrograph slit is vaguely seen in some of them, for which we have corrected implicitly by estimating min-max brightness temperature variations from the rms variations instead of taking the measured excursions. The heliograms also differ in quality; in particular, the one taken $199 \mathrm{~s}$ after launch appears somewhat sharper 
than the others (Fig. 3 of Paper I). Presumably, pointing instabilities or rocket vibrations have affected the latter in varying degree.

The left-hand end of the mean curve in the second panel of Figure 3 shows an average internetwork increase of the brightness variability $\sigma T_{b}$ of order $10 \mathrm{~K}$ over the small-scale noise limit indicated by the right-hand end. The few-degree amplitudes of the averaged modulations seen in Figures 6 and 8 are tiny with respect to the temporal and spatial variations per pixel, but they are substantial within this averaged variability and clearly significant within their variances. Thus, our spatial averaging over specific phase delay bins and jet-geometry bins in these figures brings out significant average correlations that otherwise drown in noise. The combination of small amplitudes and small variances suggests that most noise actually results from interference between intrinsic solar variations.

Considerable solar interference is undoubtedly present. If our relationships follow from regular oscillatory behavior such as the chromospheric 3 minute oscillation, phase mixing occurs because we do not Fourier-decompose the different temporal and spatial frequencies in our plots. The so-called chromospheric 3 minute oscillation is a broadband phenomenon largely made up by extended $(k, \omega)$ power ridges (e.g., Deubner et al. 1994; Hofmann, Steffens, \& Deubner 1995). Such wide temporal and spatial bandwidths dilute the amplitudes of the temporal modulations for the phase-fixed extreme Dopplershift bins in Figures 6-8, and of the spatial modulations seen in Figures 9-10. In addition, the larger scale brightness modulation with about 3-5 minute periodicity found by Cook \& Ewing (1991) by concentrating on the background $160 \mathrm{~nm}$ continuum that underlies the bright points indicates a substantial contribution from the 5 minute $p$-modes.

Solar interference may also be generated by the juxtaposition of nonsinusoidal wave modes. The disparity between blue jet geometry and red set geometry in Figures 8 and 9 indicates more complex wave forms, as does the asymmetry of the distributions in Figure 5. This is again reminiscent of the formation of $\mathrm{Ca} \mathrm{II} \mathrm{K}_{2 V}$ grains, in which vertical interference between upward traveling shocks and postshock downfall causes large disparity between $\mathrm{K}_{2 V}$ and $\mathrm{K}_{2 R}$ behavior.

Finally, it is possible that C I Dopplershift and $160 \mathrm{~nm}$ brightness are related with longer phase delays than our total data duration, or with additional phase scrambling from varying delays. Both occur in the formation of the $\mathrm{Ca}$ II $\mathrm{K}_{2 V}$ grains for which there is considerable delay between the driving Doppler excursions in the photosphere and much variation in the subsequent chromospheric grain patterns (Carlsson \& Stein 1994; cf. Rutten 1995).

\subsection{I Line Formation}

So far, we have restricted ourselves to the observational terminology of "Dopplershift" and "brightness" rather than velocity and temperature. Interpretation requires discussion in the latter terms and therefore a closer look at $\mathrm{C}_{\mathrm{I}}$ line formation. In their initial paper on jets. Dere et al. (1983) show a plot of C I line profiles. These display distinctive large-amplitude peaks that are shifted considerably from the rest wavelengths and have more or less Gaussian shapes, apart from the multiplet blending. Dere et al. (1983) argued that the observed absence of self-reversals in the peaks permits optically thin interpretation, although the $\mathrm{C}_{\mathrm{I}}$ lines are optically thick in the standard modeling by Shine, Lites, \& Chipman (1978). The latter authors display OSO 8 data with self-reversed $C_{\mathrm{I}}$ line cores possessing central dips, especially for the multiplet near $\lambda=165.7 \mathrm{~nm}$ but also for the stronger two of the three distinct lines made up by the five multiplet components near $\lambda=156.1 \mathrm{~nm}$. In addition, all lines reach about the same peak intensity. In contrast, the blue-jet peaks in Figure 3 of Dere et al. (1983) reach much higher intensities than the background nonjet profiles shown in the same figure and display increasing peak intensity and increasing Dopplershift for larger line strength. This behavior indicates lack of the saturation shown by the three background profiles. Thus, at first sight the blue-jet peaks indeed behave as optically thin high-intensity features.

Optically thin interpretation permits direct conversion of $C_{\text {I }}$ peak Dopplershifts into vertical velocities. The blue-jet values of $v \approx 10-20 \mathrm{~km} \mathrm{~s}^{-1}$ led Dere et al. (1983) to tentatively identify jets with spicules, the more so since no other EUV spicule candidate was found. Dere et al. (1986) did no longer suggest spicules, but suggested a phenomenon related to $160 \mathrm{~nm}$ bright points and internetwork $\mathrm{Ca}$ II $\mathrm{K}_{2 V}$ grains.

Our result that the $\mathrm{C}$ I lines indeed display internetwork dynamics reminiscent of $\mathrm{K}_{2 V}$ grain formation requires comparison with the formation of $\mathrm{Ca} \mathrm{II} \mathrm{H}$ and $\mathrm{K}$. The $\mathrm{C}$ I 156 $\mathrm{nm}$ lines are 1-2 orders of magnitude more opaque than $\mathrm{Ca}$ II $\mathrm{H}$ and K. The source function plot in Figure 4 of Shine et al. (1978) shows thermalization to appreciably larger height than in comparable $\mathrm{Ca}$ II $\mathrm{K}$ modeling, producing high twin peaks in the resulting line profiles (their Fig. 5). This implies that the Dopplershifts of the observed C I peaks may not simply encode motions in optically thin fashion and suggests a closer look at $\mathrm{Ca}$ II $\mathrm{K}_{2 V}$ peak formation.

In the case of the $\mathrm{H}$ and $\mathrm{K}$ lines, the classical explanation of line-core emission features as source function coupling to the chromospheric temperature rise has, for the internetwork regime, been replaced by the dynamical modeling of Carlsson \& Stein (1994). They even doubt the existence of a mean internetwork chromospheric temperature rise in the layers sampled by Ca II K (Carlsson \& Stein 1995). Instead, the internetwork chromosphere is filled with upward-traveling shocks of which the ultraviolet emission is erroneously interpreted in empirical VALIII-style modeling as mean chromospheric temperature rise. The bright $\mathrm{H}_{2 V}$ grains arise from vertical patterning of upcoming fresh shocks and backfalling matter behind preceding shocks, with much interference and temporal variation. The patterns are set by the vertical motions of the underlying photosphere, but with 5-15 minute delays between the photospheric pistoning and the resulting $\mathrm{Ca}$ II response.

The parameter diagrams in Figures 8-12 of Carlsson \& Stein (1994) are of particular interest here because these detail $\mathrm{H}_{2 V}$ grain formation in the form of breakdown snapshots. Figures 10-12 of Carlsson \& Stein (1994) show bright $\mathrm{H}_{2 V}$ peaks at apparent Dopplershift $\Delta \lambda_{\mathrm{D}} \approx 10 \mathrm{~km} \mathrm{~s}^{-1}$ that is twice the actual gas velocity at the height of peak formation. This large skewing results from the steep velocity gradients across the shocks higher up. Thus, at the height of $\mathrm{K}_{2 V}$ intensity formation, shock dynamics not only causes the striking prominence of $\mathrm{H}_{2 V}$ peaks over $\mathrm{H}_{2 R}$ peaks, but also produces doubling of the apparent peak Dopplershift over the intrinsic local Dopplershift. 
How do the $\mathrm{C}$ I peaks measured as chromospheric jets with HRTS compare to the $\mathrm{Ca} \mathrm{II}_{2 V}$ peaks in the CarlssonStein simulation? The blueshifted C I peaks in Figure 3 of Dere et al. (1983) are qualitatively similar to the $\mathrm{H}_{2 V}$ peaks in Figures 11 and 12 of Carlsson \& Stein (1994). The C I lines differ in lacking a much wider absorption profile as shown by $\mathrm{Ca}$ II $\mathrm{H}$, but this difference is due to the formation of the background continuum. The peaks have similar lifetimes, similar 2-4 minute periodicity, are similarly asymmetric with longer tails toward line center, vary similarly in apparent Dopplershift and vary strongly in amplitude in both cases. Thus, it seems that $\mathrm{C}$ I internetwork peak formation resembles Ca II $\mathrm{K}_{2 V}$ internetwork peak formation sufficiently closely that the Carlsson \& Stein simulations should be taken as interpretative guide.

\subsection{Vertical Patterning}

The $\mathrm{Ca}$ II $\mathrm{H}_{2 V}$ peaks in the Carlsson-Stein diagrams form at heights at which the shocks have just started to develop, have small strength and are yet below the height of frequent shock overtaking. The $\mathrm{H}_{2 V}$ grains therefore display fairly regular modulation in the smulated spectral evolution diagrams (Figs. 12-13 of Carlsson \& Stein). Comparable C I line formation at larger height should not only suffer from much larger shock amplitudes but also from much more confusion due to shock interference. Similarly, there should be less clear correlation with the intensity modulation in deeper layers. The Carlsson-Stein evolution diagrams display well correlated behavior in the inner wings which closely reproduces the observed $\mathrm{Ca}$ II wing "whisker modulation" observed by Beckers \& Artzner (1974). For a larger height separation between line-center formation and line-wing formation, the correlation will not be as good.

Translation to the $160 \mathrm{~nm}$ diagnostics studied here implies that $\mathrm{C}_{\mathrm{I}}$ jets represent $\mathrm{C}_{\mathrm{I}}$ profile peaks that are comparable to $\mathrm{K}_{2 V}$ peaks but are formed at larger height in the atmosphere, whereas the $160 \mathrm{~nm}$ brightness variation is comparable to the $\mathrm{Ca}$ II inner-wing whisker modulation. This seems a likely proposition. The larger solar confusion that is apparent from our data should then result from the larger amount of interference variations between shocks at larger height. The large phase difference between C I Dopplershift modulation and $160 \mathrm{~nm}$ brightness modulation, larger than $\mathrm{K}_{2 V}$-whisker phase differences, may also result from the larger $\mathrm{C}_{\mathrm{I}}$ formation height. The differences between blue and red jets should arise similarly to the differences between $\mathrm{K}_{2 V}$ and $\mathrm{K}_{2 R}$ peaks. The actual gas motions are then only a fraction of the measured C I Dopplershifts, comparable to the factor of skewing in $\mathrm{K}_{2 V}$ peak location.

\subsection{Horizontal Patterning}

The observed horizontal scalings cannot be explained from the Carlsson-Stein simulations since the latter are onedimensional. Our data confirm the split between two characteristic $160 \mathrm{~nm}$ scales observed by Foing \& Bonnet (1984a, 1984b), Foing et al. (1986), and Cook \& Ewing (1991) into mesoscale patches of 3-7 Mm and 1.4-3 Mm separation between bright points, as evident in Figures 2 and 8-9. A similar split is seen for C I Dopplershift in Figures 1 and 10.

The mesoscale patterning is also observed in Ca II $\mathrm{K}_{2 V}$ from internetwork regions. It is presumably set by the internetwork 3 minute waves and shock dynamics discussed above (Cram 1978; Cram \& Damé 1983; Damé \& Martić
1987; Lites, Rutten, \& Kalkofen 1993; cf. Rutten \& Uitenbroek 1991a).

The small-scale patterning is puzzling. In a conference presentation of our data, Hoekzema (1994) speculated that the 3 minute buffeting of a thermal interface or steep gradient between lower and upper internetwork chromosphere, possibly marking the magnetic canopy, excites horizontally spreading 3 minute surface waves that cause the observed small-scale structuring. He suggested that the 1-2 Mm separations which bright points and $\mathrm{C} I$ jets favor in Figures 8-10 are due to modulation by waves with $f$-mode character as studied theoretically by Campbell \& Roberts (1989), Evans \& Roberts (1990), and Rosenthal \& Gough (1994). This spatial scale corresponds to $f$-mode wavelengths for periodicities near 3 minutes in the low chromosphere (1.2$1.6 \mathrm{Mm}$ ).

The internetwork shocks are bound to excite surface waves with 3-minute periodicity along any discontinuity (such as a magnetic canopy). Adding their velocity amplitude to the vertical shock dynamics causes small-scale modulation of the mesoscale shock patterning. Extreme Dopplershifts may then arise from constructive interference, describing motions that are less supersonic as seen by the gas itself. In addition, such surface-wave modulation may also explain the observed small size of the jet centers, the steep amplitude drop around them, the apparent jet-center splitting, and it may also cause the observed black-nearblue areas within jets by confusing the solar C I profiles through destructive interference. The striking difference in morphology in the lower left panel of Figure 8 between fast and slow modulation decay may mark superpositions of crossing surface wave fronts, causing slender, elongated ridges of large velocity. The contrast between blue-jet and red-jet perimeter variability in the lower right panel of Figure 9 may be due to compression and rarefaction from destructive, respectively constructive, interference between the mesoscale and $f$-mode excursions. Finally, such interference may also cause the frequently stringlike appearance of the $160 \mathrm{~nm}$ bright point patterning.

\section{CONCLUSION}

We have found oscillatory relationships between C I Dopplershift variations and $160 \mathrm{~nm}$ intensity variations in chromospheric internetwork areas that seem related to the acoustic shock dynamics causing $\mathrm{Ca}$ II $\mathrm{K}_{2 V}$ grains. They indicate that C I "jets" and $160 \mathrm{~nm}$ " bright points" are not the isolated features that these names suggest, but rather the extremes of more ubiquitous oscillation patterns. The relations surface only after extensive spatial averaging, which indicates the presence of much solar variation in behavior, scales, and phases. This increase in complexity over $\mathrm{H}$ and $\mathrm{K}$ behavior may be ascribed to larger variations in shock amplitudes and shock interference patterns at larger height. In addition, there is evidence of horizontal 3 minute modulation at 1-2 $\mathrm{Mm}$ wavelength for which localized surface waves with $f$-mode character seem a likely candidate.

We have taken advantage of the absence of seeing-caused spatial deformations in the HRTS data through our spatial averaging over specific pixel classes. In the meantime, the SUMER spectrometer onboard the SOHO mission has become available. It provides excellent opportunities to study internetwork dynamics with much longer duration and with better statistics than is possible from rocket instruments, in particular enabling temporal and spatial Fourier 
decomposition and more refined phase difference and coherency studies. SOHO observing programs with simultaneous ground-based $\mathrm{Ca}$ II $\mathrm{K}_{2 V}$ imaging and spectrometry are presently underway.

With regard to interpretation, numerical simulation of dynamical C I line formation and phase relationships with $160 \mathrm{~nm}$ brightness in Carlsson-Stein fashion will be worthwhile. The C I lines and similar ultraviolet lines provide an important diagnostic step up from $\mathrm{Ca}$ II $\mathrm{H}$ and $\mathrm{K}$, to chromospheric heights where shock dynamics fully dominates. Obvious targets for reproduction are the counterphase patterns in Figures 6 and 7.

Finally, we wish to emphasize that our correlations only hold statistically, after extensive spatial averaging.
Although jets and bright points are part of oscillatory phenomena which we attribute to acoustic shock dynamics, there is so much solar interference that direct feature-byfeature linkage as in Paper I is not a good strategy. Future studies of chromospheric internetwork dynamics, whether based on SUMER data or on numerical simulations, must recognize and disentangle this intrinsic solar variety.

N. M. H.'s research was supported by the Netherlands Foundation for Research in Astronomy (NFRA) with financial aid from the Netherlands Organization for Scientific Research (NWO). J. W. C.'s work was supported by NASA under DPR W-14,541 and by the Office of Naval Research.

\section{REFERENCES}

Beckers, J. M., \& Artzner, G. 1974, Sol. Phys., 37, 309

Bonnet, R. M., Bruner, M., Acton, L. W., Brown, W. A., Decaudin, M., \& Foing, B. 1982, A\&A, 111, 125

Campbell, W. R., \& Roberts, B. 1989, ApJ, 338, 538

Carlsson, M., \& Stein, R. F. 1994, in Proc. Miniworkshop, Chromospheric

Dynamics, ed. M. Carlsson (Oslo: Inst. Theoret. Astrophys), 47 . 1995, ApJ, 440, L29

Cook, J. W., Brueckner, G. E., \& Bartoe, J.-D. F. 1983, ApJ, 270, L89

Cook, J. W., \& Ewing, J. A. 1991, ApJ, 371, 804

Cook, J. W., Rutten, R. J., \& Hoekzema, N. M. 1996, ApJ, 470, 647 (Paper I)

Cram, L. E. 1978, A\&A, 70, 345

Cram, K. E., \& Damé, L. 1983, ApJ, 272, 355

Damé, L., \& Martić, M. 1987, ApJ, 314, L15

Dere, K. P., Bartloe, J.-D. F., \& Bruecker, G. E. 1983, ApJ, 267, L65 1986, ApJ, 305, 947

Deubner, F.-L., Hoffmann, J., Kossack, E., \& Fleck, B. 1994, in Solar Surface Magnetism, NATO ASI Ser. C433, ed. R. J. Rutten \& C. J. Schrijver (Dordrecht: Kluwer), p. 155

Evans, D. J., \& Roberts, B. 1990, ApJ, 356, 704

Foing, B., \& Bonnet, R. M. 1984a, A\&A, 136, 133

.1984b, ApJ, 279, 848
Foing, B., Bonnet, R.-M., \& Bruner, M. 1986, A\&A, 162, 292

Hoekzema, N. M. 1994, in Proc. Miniworkshop, Chromospheric Dynamics, ed. M. Carlsson (Oslo: Inst. Theoret. Astrophys) 111

Hoffmann, J., Steffens, S., \& Deubner, F.-L. 1995, A\&A, 308, 192

Lites, B. W., Rutten, R. J., \& Kalkofen, W. 1993, ApJ, 414, 345

Martić, M., \& Damé, L. 1989, in Solar and Stellar Granulation, NATO ASI Ser. C 263, ed. R. J. Rutten \& G. Severino (Dordrecht: Kluwer), 207

Martić, M., Damé, L., Bruner, M. E., \& Foing, B. H. 1991, Adv. Space Res., $11,(5) 241$

Rosenthal, C. S., \& Gough, D. O. 1994, ApJ, 423, 488

Rutten, R. J. 1994, in Proc. Miniworkshop, Chromospheric Dynamics, ed. M. Carlsson (Oslo: Inst. Theor. Astrophys), 25

. 1995, in Proc. 4th SOHO Workshop, Helioseismology, ed. J. T. Hoeksema, V. Domingo, B. Fleck, \& B. Battrick, ESA SP-376 Vol. 1, ESA Publ. Div., (Noordwijk: ESTEC), 151

Rutten, R. J., \& Uitenbroek, H. 1991a, Sol. Phys., 134, 15

. 1991b, in Proc. Heidelberg Conf., Mechanisms of Chromospheric and Coronal Heating, ed. P. Ulmschneider, E. R. Priest, \& R. Rosner (Berlin: Springer), 48

Shine, R. A., Lites, B. W., \& Chipman, E. G. 1978, ApJ, 224, 247

Vernazza, J. E., Avrett, E. H., \& Loeser, R. 1981, ApJS, 45, 635 